\title{
The Case for Antenna Cancellation for Scalable Full-Duplex Wireless Communications
}

\author{
Mohammad A. \\ Khojastepour \\ NEC Laboratories America \\ Princeton, NJ \\ amir@nec-labs.com
}

\author{
Xinyu Zhang \\ University of Michigan \\ Ann Arbor, Ml \\ xyzhang@eecs.umich.edu
}

\author{
Karthik Sundaresan \\ NEC Laboratories America \\ Princeton, NJ \\ karthiks@nec-labs.com
}

\author{
Sampath Rangarajan \\ Princeton, NJ \\ sampath@nec-labs.com
}

\begin{abstract}
Recent works have considered the feasibility of full duplex (FD) wireless communications in practice. While the first FD system by Choi et.al. relied on a specific antenna cancellation technique to achieve a significant portion of selfinterference cancellation, the various limitations of this technique prompted latter works to move away from antenna cancellation and rely on analog cancellation achieved through channel estimation. However, the latter systems in turn require the use of variable attenuator and delay elements that need to be automatically tuned to compensate for the selfinterference channel. This not only adds complexity to the overall system but also makes the performance sensitive to wide-band channels. More importantly, none of the existing FD schemes can be readily scaled to MIMO systems.

In this context, we revisit the role of antenna cancellation in FD communications and show that it has more potential in its applicability to FD than previously thought. We advocate a design that overcomes the limitations that have been pointed out in the literature. We then extend this to a twostage design that allows both transmit and receive versions of antenna cancellation to be jointly leveraged. Finally, we illustrate an extension of our design to MIMO systems, where a combination of both MIMO and FD can be realized in tandem.
\end{abstract}

\section{Categories and Subject Descriptors}

C.2.1 [Network Architecture and Design]: Wireless Communication

Permission to make digital or hard copies of all or part of this work for personal or classroom use is granted without fee provided that copies are not made or distributed for profit or commercial advantage and that copies bear this notice and the full citation on the first page. To copy otherwise, to republish, to post on servers or to redistribute to lists, requires prior specific permission and/or a fee.

Hotnets '11, November 14-15, 2011, Cambridge, MA, USA.

Copyright 2011 ACM 978-1-4503-1059-8/11/11 ...\$10.00.

\section{General Terms}

Design, Performance

\author{
Sanaz Barghi \\ University of California \\ Irvine, $\mathrm{CA}$ \\ sbarghi@uci.edu
}

\section{INTRODUCTION}

A full-duplex wireless device that can transmit and receive at the same time in the same frequency band by definition would need at least one Tx and one Rx antenna. The key challenge in realizing such a device lies in addressing the self-interference generated by the Tx antenna at the Rx antenna. As an example, consider a WiFi signal with a transmit power of $20 \mathrm{dBm}$. A Tx-Rx antenna separation of about 68 inches results in a path loss of about $40 \mathrm{dBm}$ (depending on channel characteristics), resulting in a self-interference of at least $-20 \mathrm{dBm}$. With a noise floor around $-93 \mathrm{dBm}$, one would further require a self-interference cancellation of at least $73 \mathrm{~dB}$ to be able to decode the desired received signal. While one can solely employ digital interference cancellation techniques, current ADC's do not have a resolution to pass a received signal which is $73 \mathrm{~dB}$ less than the noise floor. Hence, several practical full duplex (FD) systems [1, $2,3]$ have been proposed that couple RF cancellation along with digital cancellation to achieve the desired level of selfinterference suppression.

Choi et. al. [1] proposed an architecture that used a combination of RF cancellation and digital cancellation techniques. RF cancellation included both antenna as well as analog cancellation (using noise cancellation circuits), contributing around $30 \mathrm{~dB}$ and $20 \mathrm{~dB}$ of cancellation respectively. With an additional $10 \mathrm{~dB}$ from digital cancellation, this resulted in a total of $60 \mathrm{~dB}$ suppression. Although not sufficient for $\mathrm{WiFi}$, this was sufficient to enable FD communication in 802.15.4 systems (with $0 \mathrm{dBm}$ transmit power). Antenna cancellation was achieved with the help of two Tx antennas being placed at $\ell$ and $\ell+\frac{\lambda}{2}$ distance from the $\mathrm{Rx}$ antenna. The $\frac{\lambda}{2}$ adds a phase shift of $\pi$ to one of the transmitted signals to help cancel the other transmitted signal at the Rx antenna. Three limitations of such an antenna cancel- 
lation approach were pointed out by the same authors in [2]: (i) the dependence on $\lambda$ allows for maximum cancellation only at the center frequency, with performance degrading for frequencies away from the center - a problem for wideband systems; (ii) employing an additional antenna may not justify the gains compared to a $3 \times 3$ MIMO system, and (iii) due to asymmetric antenna placement, manual tuning of amplitude and phase of the closer Tx antenna is required to achieve a null, which prevents real-time operation.

To avoid the above limitations, the authors moved away from antenna cancellation and instead proposed the use of a two antenna (one Tx, one Rx) scheme [2], where a form of analog (BALUN - balanced to unbalanced transformer) cancellation was used. A BALUN element acts as a $\pi$ phase shifter, which was shown to have a better frequency response over a wideband compared to the $\lambda$ dependent phase shift created using an asymmetric antenna placement. BALUN cancellation was shown to yield $40-45 \mathrm{~dB}$ of cancellation; this coupled with $30 \mathrm{~dB}$ cancellation using digital cancellation provided the desired level of self-interference suppression for WiFi signals. However, such a design encounters the following limitation. While a BALUN element can create a negative copy of the transmitted signal that can be applied internally to cancel self-interference, one also needs to account for the wireless channel between the Tx and Rx antennas. For this reason, a variable attenuator and delay element are also needed on the path, which in turn have to be auto-tuned and adapted to track the self-interference channel. This not only makes the design complicated but also the performance quite sensitive to wide-band channels. Although with manual tuning it is shown that $40-45 \mathrm{~dB}$ cancellation could be achieved, in practice, auto-tuning leads to only a $20 \mathrm{~dB}$ cancellation [2]. Other works on FD implementations such as Duarte et. al. [3] and Sahai et. al. [4] also do not consider antenna cancellation but consider hybrid schemes where an estimate of the self-interference signal in the digital domain is combined with a negated copy of the transmitted signal in the analog domain to achieve cancellation. This along with digital cancellation was shown to yield only about $35 \mathrm{~dB}$ of cancellation, falling short of the desired target.

Next generation wireless devices (access points, base stations, etc.) are expected to be equipped with multiple antennas (more than two). Hence, it is important to design a FD scheme that can co-exist with MIMO. We observe that existing antenna cancellation [1] and analog cancellation [2] approaches cannot be readily extended to MIMO systems. Although one might envision an extension of [1] using two Tx and one Rx antenna for every transmitted/received MIMO stream, this would require antennas to be placed such that each of the Tx pairs (for each stream) lead to self-interference signals which are $180^{\circ}$ out of phase at every $\mathrm{Rx}$ antenna. However, such an antenna placement cannot be realized for a MIMO system using the approach in [1] (discussed in Sec 2 ). On the other hand, analog cancellation in [2], when extended to $N$ stream MIMO, will potentially require one to estimate the self-interference channel between every pair of

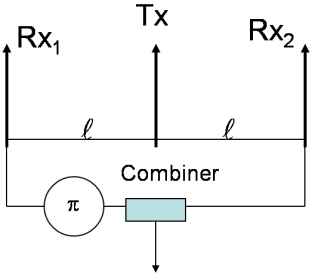

a) Receive Cancellation

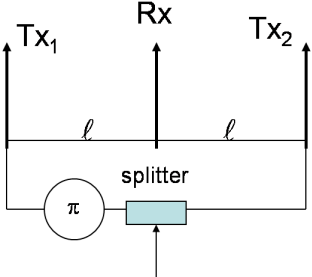

b) Transmit Cancellation
Figure 1: Antenna Cancellation.

$N^{2}$ Tx-Rx antennas. This in turn results in the use of $N^{2}$ variable delays and attenuators, each of which has to be auto tuned and adapted to track the $N^{2}$ self-interference channels, which seems practically infeasible.

Based on our observations on the limitations of existing FD schemes, we propose and justify antenna cancellation with symmetric placement of antennas be considered as a primary RF cancellation technique. Specifically, for a single stream transmission we propose antenna cancellation with a symmetric placement of either two Rx antennas and one Tx antenna (which we refer to as Rx antenna cancellation), or two Tx antennas and one Rx antenna (which we refer to as Tx antenna cancellation), each of which is a dual of the other. We show that this design could provide large selfinterference cancellation with the following advantages:

(a) It leads to the possibility of a two-level design where Tx antenna cancellation is followed by a Rx antenna cancellation with the theoretical potential to double the antenna cancellation gains because of its additive nature.

(b) The design scales very easily to MIMO systems which would then enable the co-existence of MIMO with FD.

(c) The design could potentially eliminate the need for any other form of analog cancellation which seems limited in practice due to the need for variable attenuators and delay elements and its subsequent lack of scalability to MIMO systems.

Next, we discuss our proposal for a two-level antenna cancellation design and show how it could easily be extended to MIMO systems. Then, we discuss practical aspects of the proposed technique, and provide preliminary measurement results to substantiate some of our conclusions and highlight the applicability of FD in indoor and outdoor scenarios.

\section{THE CASE FOR SYMMETRIC ANTENNA PLACEMENT}

We now present our antenna cancellation approach which is based on a symmetric placement of the antennas. Figure 1(a) illustrates our Rx antenna cancellation, where two $\mathrm{Rx}$ antennas are placed symmetrically at a distance $\ell$ from the Tx antenna. The signal received from one of the receive antennas is phase shifted internally using a fixed $\pi$ phase shifter before being combined with the other receive signal to help nullify the self-interference signal. Similar to Rx antenna cancellation, we can also have an analogous $\mathrm{Tx}$ 
antenna cancellation as shown in Fig. 1(b). While the basic antenna configuration for cancellation is simple, we now highlight its significant potential to address the limitations of existing FD schemes. More importantly, we show how it can be extended to two levels of antenna cancellation and leveraged in tandem with MIMO - previously not addressed in the literature.

\subsection{Advantages of Symmetric Antenna Place- ment}

Compared to the transmit antenna cancellation in [1], where the $\pi$ phase shift was realized with asymmetric placement of Tx antennas $\left(\ell\right.$ and $\left.\ell+\frac{\lambda}{2}\right)$, our approach has the following advantages.

(a) Bandwidth Dependence: Moving the $\pi$ phase shift internally alleviates the bandwidth dependence (due to $\lambda$ ) of antenna cancellation. Further, fixed $\pi$ phase shifters have significantly better frequency responses over wide bandwidths compared to variable ones.

(b) Tuning: Since the received powers are similar, this avoids the need for tuning of attenuation and phase of the selfinterference signal; otherwise required to counteract the power difference due to asymmetric antenna placement.

Further, unlike [2], our approach does not require estimation of the self-interference channel between Tx and Rx antennas and hence the need for variable attenuators and delay lines. [2] uses a BALUN to generate a $\pi$ phase shift internally. However, in the absence of antenna cancellation, this is not sufficient and variable attenuators and delay lines or tunable RF cancellation circuits are needed to compensate for the self-interference channel.

One limitation that was raised with respect to symmetric antenna cancellation in [1], is its impact on far field signals. [1] advocated the use of asymmetric Tx antenna spacing (instead of internal phase shifter) to generate the required phase shift of $\pi$ between the transmit signals owing to its ability to cause less destructive interference in the far field. However, the simulations used to highlight this observation relied on a free space path loss model for the far field which does not hold in reality (see experiments in Section 4). While the selfinterference channel can be modeled as free space, it is well known that the far field channels (indoors or outdoors) from the transmit antennas experience indepedent (Rayleigh) fading at any far field receive point. Hence, asymmetric antenna spacing does not provide any advantage over a symmetric placement (with internal phase shifter) with respect to impact on far-field. Analogously, in our symmetric receive antenna cancellation (with an internal $\pi$ phase shifter), while the received signals from the node's own transmit antenna experiences a free space channel, it experiences independent fading when the transmit signal is generated from far field.

Comparison with MIMO: In general, antenna cancellation requires an extra antenna which [2] believed may not be justifiable compared to a $3 \times 3$ MIMO system. MIMO transmissions require each antenna to have a $\mathrm{Tx} / \mathrm{Rx} \mathrm{RF}$ chain, which is not the case here; antenna cancellation merely re-

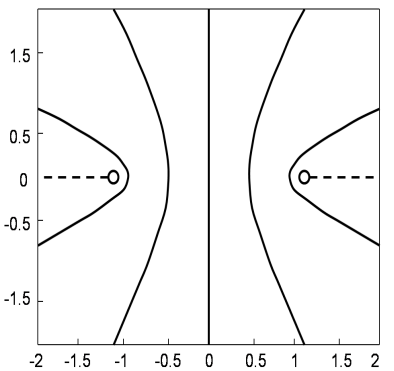

a) Phase Offset + Antenna Spacing

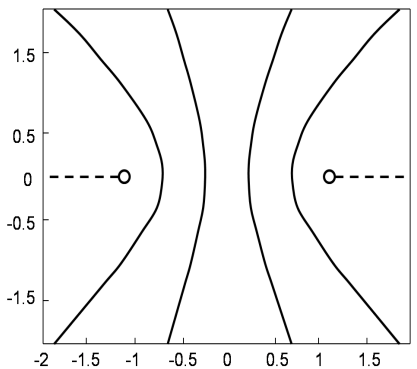

b) Only Antenna Spacing
Figure 2: Loci of Null Points.

quires an extra passive antenna element together with a fixed phase shifter and the overhead is not comparable to a MIMO system. If at all any comparison should be made, it should be with a $2 \times 2$ MIMO system (as in [2]).

We now provide a brief discussion on some of the properties pertaining to antenna cancellation, that will be leveraged in our extension to two level cancellation as well as MIMO.

\subsection{Understanding Antenna Cancellation}

To leverage antenna cancellation effectively, it is important to understand the notion of signal nulling. A signal is said to be "nulled" when two copies of the signal add $\pi$ out of phase to cancel each other, thereby pushing the received signal strength to or below the noise floor. Let us consider transmit antenna cancellation for explaining the concepts. There are two parameters affecting the nulling process: relative phase and amplitude of the transmitted signals at the receiver. The relative phase between the two signals could be further controlled either by directly introducing a phase offset $(\phi)$ to one of the signals and/or by varying the relative distance between the transmit antennas with respect to the receive antenna.

Let $d_{t}$ be the distance between the two Tx antennas, with $d_{1}$ and $d_{2}$ denoting the distance of the two transmit antennas with respect to a receive point respectively. First, we consider the set of potential receive null points, where there is a phase offset $\phi=\pi$. Whether these null points can be realized in turn depends on the relative amplitude of the signals as well, which is discussed subsequently. Now the set of potential null points in a two-dimensional plane can be defined as the locus of the points satisfying $\left|d_{1}-d_{2}\right|=k \lambda$ for some integer $k$ and includes the following (see Fig. 2(a)).

- The perpendicular bisector (PB) of the line joining the transmit antennas (i.e. $d_{1}=d_{2}=\frac{d_{t}}{2}$ ).

- A set of hyperbolas with the transmit antennas as the focal points. Each hyperbola intersects the line connecting the two transmit antennas at points that are $\frac{k \lambda}{2}, k \in \mathcal{Z}, \geq$ 1 from the mid-point towards either one of the transmit antennas.

- If $d_{t}=m \lambda$, in addition to the above points, all points on the line passing through the two transmit antennas besides those lying in between them also contribute to the set of potential null points. 


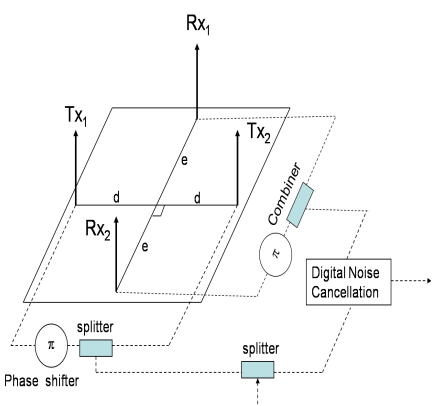

a) Two Level Placement

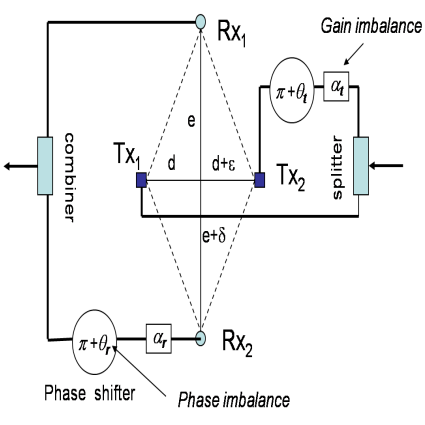

b) Imprecise Placement
Figure 3: Two Level Antenna Cancellation.

To understand scenarios where relative phase is controlled only with the help of antenna spacing (i.e. phase offset, $\phi=$ 0 ), we note that the locus of the potential null points is now defined as those satisfying $\left|d_{1}-d_{2}\right|=\frac{(2 k+1) \lambda}{2}$ and consist of (see Fig. 2(b)),

- A set of hyperbolas with the transmit antennas as the focal points. Each hyperbola intersects the line connecting the two transmit antennas at points that are $\frac{(2 k+1) \lambda}{4}$, $k \in \mathcal{Z}, \geq 1$ from the mid-point towards either one of the transmit antennas.

- If $d_{t}=\frac{(2 m+1) \dot{\lambda}}{2}$, in addition to the above points, all points on the line passing through the two transmit antenna besides those lying in between them also contribute to the set of potential null points.

Now for a potential null point to be realized, the two transmit signals must arrive at the receive point with equal amplitude but $\pi$ out of phase. Due to symmetry, this can be easily achieved on the PB with an equal transmit power from the two transmit antennas. Hence, all null points on the PB are realizable. However, for a null point on a hyperbola, it is easy to see that different transmit powers will be required from the two transmit antennas. Further, this will vary from one point to another on the same hyperbola as well as across hyperbolas. Hence, for a fixed (potentially different) transmit power from the two transmit antennas, at most two null points on each hyperbola may be realizable. Note that we do not have null points on the PB when $\phi \neq \pi$. Given that the null points on the hyperbolas are hard to realize, this limits the applicability of asymmetric antenna spacing based approaches (eg. [1]) to two level antenna cancellation; this limitation is compounded in the case of MIMO. This important property of realizing null points on the PB when transmit signals are phase shifted by $\pi$ is leveraged later for two purposes: (1) extend the transmit antenna cancellation to a two-level transmit and receive antenna cancellation scheme, and (2) to realize FD communication in tandem with MIMO.

3. TWO LEVEL CANCELLATION AND MIMO

\subsection{Two Level Antenna Cancellation}

Given that the above properties of transmit antenna cancellation (based on phase offset) analogously apply to receive cancellation as well, we can easily extend our proposed scheme to employ two stages (transmit and receive) of antenna cancellation in tandem. In the first stage two transmit antennas transmit at equal power and $\pi$ out of phase signals that destructively interfere at any point on the PB of the transmit antennas. Now, place two RX antennas symmetrically on the PB of the transmit antennas as shown in Fig. 3(a), such that the TX and RX sets of antennas are on each other's PB. While the transmit signals add destructively at each RX antenna, the signals received from the two RX antennas are further combined 180 degrees out of phase to provide the second level of antenna cancellation. Note that though four antennas are employed to achieve two levels of antenna cancellation, the number of RF chains used is still only two (for forward and reverse streams).

The isolation (in $\mathrm{dB}$ ) achieved by these two stages of cancelation are additive in theory although in practice the cancelations might not be perfectly additive. In fact under ideal conditions even a one stage cancelation should provide a perfect null. However, gain imbalance or a slight phase offset between the signals may prevent us from achieving a perfect null, wherein a residue of the self-interference signal remains. We can now establish the following property.

PROPERTY 1. Under small gain imbalance and/or phase offset (from imprecise antenna placement or imperfect $R F$ devices) between the transmit and receive cancellation paths, the self-interference cancellation provided by two levels of antenna cancellation are additive (in $\mathrm{dB}$ scale).

Proof. Consider the antenna placement in Figure 3(b). We model the imprecision in antenna placement with small deviation in distances as $d$ and $d+\epsilon$ for the 2 TX antennas on the horizontal axis, and $e$ and $e+\delta$ for the 2 RX antennas on the vertical axis $(\epsilon, \delta \ll d, e)$. We also model the imprecision in the RF circuitry by considering small phase differences $\theta_{t}$ and $\theta_{r}$ and gain differences $\alpha_{t}$ and $\alpha_{r}$ in the transmit and receive cancelation circuits, respectively. The received signal $y(t)$ at time $t$ can now be written as

$$
\begin{aligned}
y(t) & =A_{11} x(t) e^{j\left(2 \pi f_{c} t+\phi_{11}\right)}+A_{12} x(t) e^{j\left(2 \pi f_{c} t+\phi_{12}\right)} \\
& +A_{21} x(t) e^{j\left(2 \pi f_{c} t+\phi_{21}\right)}+A_{22} x(t) e^{j\left(2 \pi f_{c} t+\phi_{22}\right)}
\end{aligned}
$$

where $x(t)$ is the baseband signal, $f_{c}$ is the transmission frequency, and $A_{i j}$ and $\phi_{i j}$ denotes the gain and phase shift of the signal transmitted from transmit antenna $i$ to receive antenna $j$ under free space path loss model. Note that $A_{i j}$ and $\phi_{i j}$ include the gain imbalance and phase imprecision caused by RF circuitry as well as imprecise placement of the antennas. Let $d_{i j}$ denote the distance between transmit antenna $i$ to the receive antenna $j$.

$$
\begin{aligned}
& \text { We have, } d_{11}=\sqrt{d^{2}+e^{2}}, d_{12} \approx d_{11}+\frac{e}{d_{11}} \delta \\
& d_{21} \approx d_{11}+\frac{d}{d_{11}} \epsilon, d_{22} \approx d_{11}+\frac{d}{d_{11}} \epsilon+\frac{e}{d_{11}} \delta
\end{aligned}
$$

For gain, we have, $A_{11}=A\left(\frac{4 \pi \lambda}{d_{11}}\right)^{2}, A_{12}=A \alpha_{r}\left(\frac{4 \pi \lambda}{d_{12}}\right)^{2}=A_{11} a_{12}$,

$$
\text { where, } a_{12}=\alpha_{r}\left(\frac{d_{11}}{d_{12}}\right)^{2} \approx \alpha_{r}\left(1-\frac{2 e \delta}{d_{11}^{2}}\right)
$$




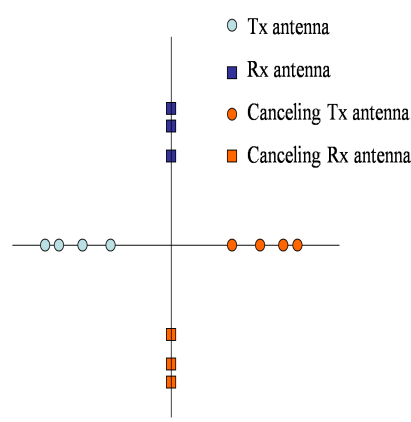

a) 2 Level AC + MIMO (4x3)

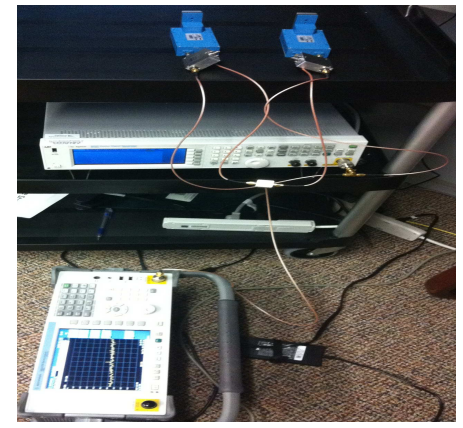

b) Receive Cancellation Expt
Figure 4: Extension to MIMO; Expt Set-up

Similarly, $A_{21}=A \alpha_{t}\left(\frac{4 \pi \lambda}{d_{21}}\right)^{2}=A_{11} a_{21} \approx A_{11} \alpha_{t}\left(1-\frac{2 d \epsilon}{d_{11}^{2}}\right)$ Now, it can be shown that, $A_{22}=A \alpha_{t} \alpha_{r}\left(\frac{4 \pi \lambda}{d_{22}}\right)^{2} \approx A_{11} a_{12} a_{21}$ For phase, $\phi_{11}=\frac{2 \pi d_{11}}{\lambda}, \phi_{12}=\left(\pi+\theta_{r}\right)+\frac{2 \pi d_{12}}{\lambda}=\pi+\phi_{11}+\xi_{12}$

$$
\phi_{21}=\left(\pi+\theta_{t}\right)+\frac{2 \pi d_{21}}{\lambda}=\pi+\phi_{11}+\xi_{21}
$$

We now have, $\phi_{22}=\left(\pi+\theta_{t}\right)+\left(\pi+\theta_{r}\right)+\frac{2 \pi d_{22}}{\lambda}=\phi_{11}+\xi_{12}+\xi_{21}$

In one-stage receive antenna cancelation (TX2 is not transmitting), the received signal can be manipulated as

$$
\begin{aligned}
y_{1}(t) & \approx A_{11} x(t) e^{j\left(2 \pi f_{c} t+\phi_{11}\right)}\left(1-e^{j \xi_{12}}\right) \\
& +A_{11}\left(a_{12}-1\right) x(t) e^{j\left(2 \pi f_{c} t+\phi_{12}\right)}
\end{aligned}
$$

Similarly, in one-stage transmit antenna cancelation (RX2 is not receiving), the received signal can be manipulated as

$$
\begin{aligned}
y_{2}(t) & \approx A_{11} x(t) e^{j\left(2 \pi f_{c} t+\phi_{11}\right)}\left(1-e^{j \xi_{21}}\right) \\
& +A_{11}\left(a_{21}-1\right) x(t) e^{j\left(2 \pi f_{c} t+\phi_{21}\right)}
\end{aligned}
$$

For our two-stage cancelation, we can denote

$$
\begin{aligned}
y(t) & \approx A_{11} x(t) e^{j\left(2 \pi f_{c} t+\phi_{11}\right)}\left(1-e^{j \xi_{12}}\right) \\
& +A_{11}\left(a_{12}-1\right) x(t) e^{j\left(2 \pi f_{c} t+\phi_{12}\right)} \\
& +A_{21} x(t) e^{j\left(2 \pi f_{c} t+\phi_{21}\right)}\left(1-e^{j \xi_{12}}\right) \\
& +A_{21}\left(a_{12}-1\right) x(t) e^{j\left(2 \pi f_{c} t+\phi_{22}\right)} \\
& \approx A_{11} x(t) e^{j\left(2 \pi f_{c} t+\phi_{11}\right)}\left(1-e^{j \xi_{12}}\right)\left(1-e^{j \xi_{21}}\right) \\
& +A_{11}\left(a_{12}-1\right) x(t) e^{j\left(2 \pi f_{c} t+\phi_{12}\right)}\left(1-e^{j \xi_{21}}\right) \\
& +A_{11}\left(a_{21}-1\right) x(t) e^{j\left(2 \pi f_{c} t+\phi_{21}\right)}\left(1-e^{j \xi_{12}}\right) \\
& +A_{11}\left(a_{12}-1\right)\left(a_{21}-1\right) x(t) e^{j\left(2 \pi f_{c} t+\phi_{22}\right)}
\end{aligned}
$$

We have $\frac{y(t)}{\tilde{x}(t)} \approx \frac{y_{1}(t)}{\tilde{x}(t)} \cdot \frac{y_{2}(t)}{\tilde{x}(t)}$, where $\tilde{x}(t)=A_{11} x(t) e^{j\left(2 \pi f_{c} t+\phi_{11}\right)}$, implying that the gains of the two stages are additive.

\subsection{Extension to MIMO Systems}

Realizing null points on a straight line is critical because it facilitates the design of MIMO transmit and receive antenna arrays. Hence, our proposed two-level antenna cancellation solution based on phase offset can be readily extended to MIMO systems by using ordinary and widely used linear antenna array configurations. In particular, to generate a $N \times M$ MIMO+FD system, we start by placing two sets of antennas ( $N$ transmit and $M$ receive) on two perpendicular axis to allow for $N \times M$ MIMO (in each direction of FD) as shown in Fig. 4(a) $((N, M)=(4,3))$. Then, to enable this $N \times M$ system with full duplex, we use an equal number of transmit $(N)$ and receive $(M)$ canceling antennas and place them in a symmetric position on the opposite side of their respective axis. The MIMO transmit streams from the $N$ transmit and their respective cancelling antennas will add out of phase at each of the receive antennas in the first stage of cancellation. The composite received signals at each of the $M$ receive antennas are then further combined out of phase with their respective cancelling antennas to provide the second level of cancellation. It is worth pointing out that only such symmetric antenna configurations can be extended to generic MIMO systems without the need for variable attenuators and delay elements. Again note that, while $2(N+M)$ antennas are employed for achieving two levels of antenna cancellation with FD, the total number of RF chains required is only $N+M$, which is the minimum required to enable $N \times M$ MIMO communication in either direction. Fig. 4(a) shows the antenna structure for a $4 \times 3$ MIMO+FD node.

\subsection{Comparison with Analog Cancellation}

The fixed phase offset of $\pi$ between copies of the transmit (receive) signals in our design can be accomplished with the help of a splitter (combiner) and a fixed $\pi$ phase shifter. Note that the insertion loss of the phase shifter has to be compensated in the other signal path. Alternately, the splitter (combiner) and phase shifter can be replaced by a BALUN if its frequency response is more flat over the desired band. A key feature of our design is that with two levels of antenna cancellation, we could potentially avoid analog cancellation and the consequent use of variable attenuators and delays. This is important in a practical system for several reasons: (i) estimating the self-interference channel in a wide-band frequency selective channel cannot be accomplished effectively with a single variable attentuator and delay line; (ii) a $N \times N$ MIMO system would require $N^{2}$ variable attenuators and delays, while our design requires only $N$ fixed $\pi$ phase shifters; and (iii) while our design employs only one passive RF component, variable attenuators and delays are typically realized with the help of active RF components such as voltage controlled attenuator, programable delay lines, etc. Active RF components have relatively poor frequency response in wide-bands and introduce RF active noise and hence must be limited in use or completely avoided if possible.

\section{PRACTICAL REALIZATION}

Our proposal for joint MIMO and FD system is to incorporate two levels of antenna cancellation along with digital cancellation. Prior work [1] has shown cancellation of 25$30 \mathrm{~dB}$ with asymmetric Tx antenna cancellation. While the gains from the two levels of antenna cancellation are theo- 
$\begin{array}{ll}\text { (a) Outdoor near-field, position } 1 & \text { (b) Outdoor near-field, position } 2\end{array}$

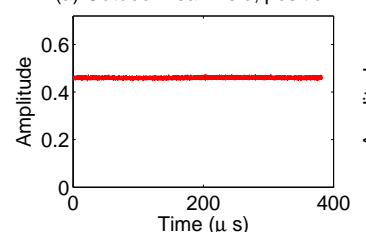

(c) Indoor near-field, position 1
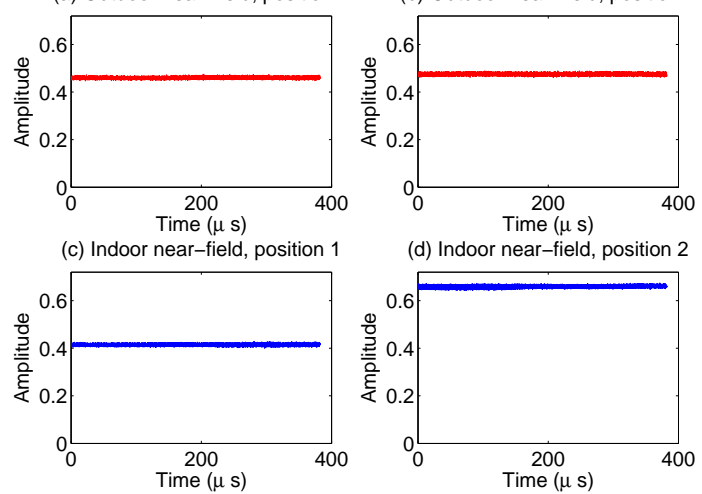

(d) Indoor near-field, position 2

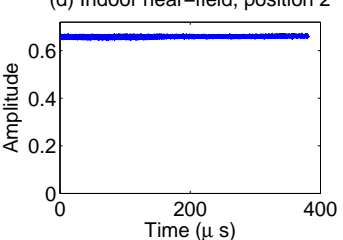

Figure 5: Near Field Experiment

retically additive in our scheme, we expect to gain over at least $50 \mathrm{~dB}$ net cancellation in practice from the two stages. Besides antenna placement, we can also leverage antenna orientation to contribute to additional self-interference suppression. Particularly, we can use +45 and -45 degree polarized antennas for transmission and reception respectively. Antenna cancellation coupled with about $20 \mathrm{~dB}$ of digital cancellation (as observed in [4,3]) along with another $10 \mathrm{~dB}$ isolation from antenna polarization will help us achieve the desired target.

Preliminary Experiments: Given that our main focus is on antenna cancellation, we have performed some preliminary experiments to verify its feasibility. Note that three assumptions are critical for our antenna cancellation to work effectively: (i) channel between a Tx and Rx antenna is symmetric across both signal and cancellation paths (dominant LOS component in near field); (ii) channel is fading in the far field; and (iii) fixed phase shifters can yield a $\pi$ phase offset for a wide-band (similar to BALUN [2]).

We verify the first assumption by performing the following experiment both indoors and outdoors. The received signal (magnitude and phase) from a transmit antenna at two symmetrically placed receive antennas were measured in both the near ( 1 feet) and far ( $3 \mathrm{~m})$ fields. We use WARP boards and perform channel estimation over a bandwidth of $625 \mathrm{KHz}$ for a duration of 400 microsecs with 16384 samples. Figs. 5(a) and (b) illustrate that while the channel magnitudes in the near-field are almost constant within the measurement resolution and error of WARP boards in outdoors, they could vary considerably indoors (Fig. 5(c) and (d)). The measured channel phase also exhibited a similar behavior.

We verify our second assumption along with polarization experiments. Using orthogonally polarized transmit and receive antennas in the near field resulted in considerable isolation of about $10 \mathrm{~dB}$ in our experiments. However, in the far field, the received channel magnitude at two different points ( $3 \mathrm{~m}$ away from transmit antenna) did not have any correlation with polarization. Figure 6 reinforces the lack of correlation between received signals in the far field, which is true in the case of polarization as well.

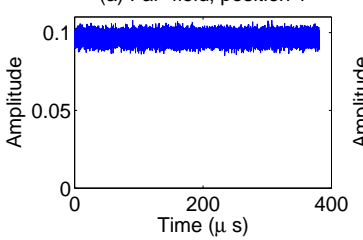

(b) Far-field, position 1, polarized

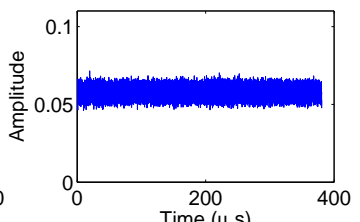

(c) Far-field, position 2

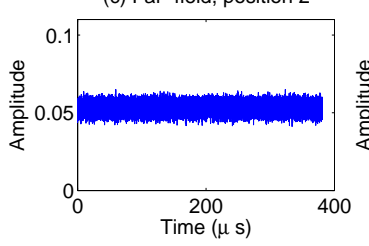

(d) Far-field, position 2, polarized

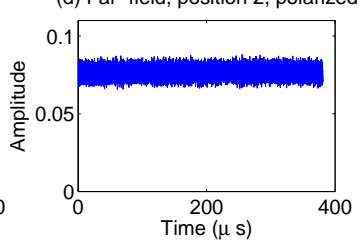

Figure 6: Far Field Experiment with Polarization

To get an estimate of the amount of RF cancellation possible with a fixed $\pi$ phase shifter, we conduct the following experiment, whose set-up is shown in Fig. 4(b). For the transmit signal, we employed both a signal generator as well as a WiMAX BS that generates a signal of $10 \mathrm{MHz}$ bandwidth, centered at $5 \mathrm{GHz}$ carrier frequency. The signal was passed through a splitter; the resulting two copies were passed through two phase shifters of 0 and $\pi$ phase shifts respectively; and then combined to observe the received signal power. Two phase shifters were intentionally used to generate similar insertion loss on the two paths. It was found that for a $0 \mathrm{dBm}$ transmit signal, one level of receive cancellation was able to yield as much as $55 \mathrm{~dB}$ suppression at the center tone and up to $45 \mathrm{~dB}$ over the $10 \mathrm{MHz}$ bandwidth.

\section{CONCLUSIONS}

We have highlighted the various limitations of existing FD schemes and their inability to scale to MIMO systems. Given that co-existence with MIMO is crucial for next generation multiple antenna devices, we propose an antenna cancellation approach that not only addresses the limitations of existing FD schemes, but also scales readily to accommodate two levels of antenna cancellation as well as MIMO. Hence, we advocate the proposed antenna cancellation as an important candidate for next generation FD systems. However, several challenges remain in implementing such a system in practice, which in turn are being explored currently.

\section{REFERENCES}

[1] J. Choi, M. Jain, K. Srinivasan, P. Levis, and S. Katti, "Achieving single channel, full duplex wireless communication," in $A C M$ MobiCom, Sept 2010.

[2] M. Jain et. al., "Practical, real-time, full duplex wireless," in ACM Mobicom 2011, Sept 2011.

[3] M. Duarte and A. Sabharwal, "Full-duplex wireless communications using off-the-shelf radios: Feasibility and first results," in Asilomar, Nov 2010.

[4] A. Sahai, G. Patel, and A. Sabharwal, "Pushing the limits of full-duplex: Design and real-time implementation," in Arxiv, July 2011. 